\title{
Proton Elastic Form Factor Ratio: the JLab Polarization Experiments
}

\author{
C.F. Perdrisat*, V. Punjabi ${ }^{\dagger}$ and the Jefferson Lab Hall A and $\mathrm{G}_{E p}(I I I)$ \\ Collaborations** \\ ${ }^{*}$ College of William and Mary, Williamsburg, VA 23187 \\ ${ }^{\dagger}$ Norfolk State University,Norfolk, VA 23504 \\ ${ }^{* *} 12000$ Jefferson Avenue, Newport News, VA 23666
}

\begin{abstract}
The ratio of the electric and magnetic proton form factors, $G_{E p} / G_{M p}$, has been obtained in two Hall A experiments, from measurements of the longitudinal and transverse polarization of the recoil proton, $P_{\ell}$ and $P_{t}$, respectively, in the elastic scattering of polarized electrons, $\vec{e} p \rightarrow e \vec{p}$. Together these experiments cover the $\mathrm{Q}^{2}$ - range 0.5 to $5.6 \mathrm{GeV}^{2}$. A new experiment is currently being prepared, to extend the $\mathrm{Q}^{2}$-range to $9 \mathrm{GeV}^{2}$ in Hall $\mathrm{C}$.
\end{abstract}

\section{INTRODUCTION}

The nucleon elastic form factors describe the internal structure of the nucleon; in the non-relativistic limit, for small four-momentum transfer squared, $\mathrm{Q}^{2}$, they are Fourier transforms of the charge and magnetization distributions in the nucleon. At high $Q^{2}$ values, the nucleon must be treated as a system of three valence quarks; perturbative QCD predicts the $\mathrm{Q}^{2}$-dependence [1] of the form factors. At $\mathrm{Q}^{2}$ between 1 and $10 \mathrm{GeV}^{2}$, relativistic constituent quark models $[2,3]$ currently give the best understanding of the nucleon form factors, with the strongest dynamical input; Vector Meson Dominance (VMD) (see e.g. Refs. [4, 5]) also describes the form factors well.

The unpolarized elastic ep cross section is given by:

$$
\frac{d \sigma}{d \Omega}=\frac{\alpha^{2} E_{e}^{\prime} \cos ^{2} \frac{\theta_{e}}{2}}{4 E_{e}^{3} \sin ^{4} \frac{\theta_{e}}{2}}\left[G_{E p}^{2}+\frac{\tau}{\varepsilon} G_{M p}^{2}\right]\left(\frac{1}{1+\tau}\right),
$$

where $G_{E p}$ and $G_{M p}$ are the electric and magnetic form factors, $\varepsilon=[1+2(1+$ $\left.\tau) \tan ^{2}\left(\frac{\theta_{e}}{2}\right)\right]^{-1}, \theta_{e}$ is the scattering angle of the electron in the laboratory and $\tau=Q^{2} / 4 M_{p}^{2}$, with $M_{p}$ the proton mass; $E_{e}$ and $E_{e}^{\prime}$ are the energies of the in- and outgoing electrons, respectively. For a given $\mathrm{Q}^{2}, G_{E p}$ and $G_{M p}$ can be extracted from cross section measurements made at fixed $\mathrm{Q}^{2}$, over a range of $\varepsilon$ values with the Rosenbluth method. At $Q^{2}$ below $1 \mathrm{GeV}^{2}, G_{E p}$ and $G_{M p}$ have been determined by this method and $\mu_{p} G_{E p} / G_{M p}$ has been found to be $\approx 1$. At larger $\mathrm{Q}^{2}$, the cross section becomes dominated by the $G_{M p}$ contribution; $G_{M p}$ is known up to $\mathrm{Q}^{2}=$ 


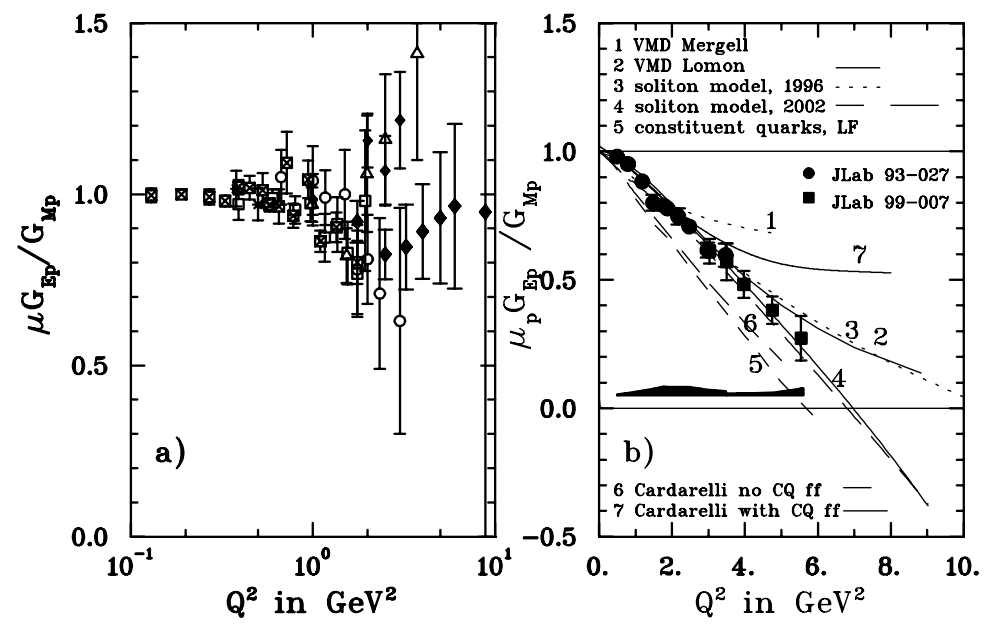

FIGURE 1. a) World data for $\mu_{p} G_{E p} / G_{M p}$ versus $\mathrm{Q}^{2}$, not including the JLab polarization data. b) the JLab data compared with several theoretical predictions. The systematic uncertainties are show as the black polygon.

$31 \mathrm{GeV}^{2}$ [6]. In Fig. 1a), the error bars on $\mu_{p} G_{E p} / G_{M p}$ from the World cross section data (refs. [7, 8, 9, 10, 11, 12, 13]) are seen to grow with $\mathrm{Q}^{2}$. Above $\mathrm{Q}^{2} \approx 1 \mathrm{GeV}^{2}$, systematic differences between different experiments are evident.

The JLab results have been obtained by measuring the recoil proton polarization in $\vec{e} p \rightarrow e \vec{p}[14,15]$. In one-photon exchange, the scattering of longitudinally polarized electrons on unpolarized hydrogen results in a transfer of polarization to the recoil proton with two components, $P_{t}$ perpendicular to, and $P_{\ell}$ parallel to the proton momentum in the scattering plane [16]:

$$
\begin{gathered}
I_{0} P_{t}=-2 \sqrt{\tau(1+\tau)} G_{E_{p}} G_{M_{p}} \tan \frac{\theta_{e}}{2} \\
I_{0} P_{\ell}=\frac{1}{M_{p}}\left(E_{e}+E_{e^{\prime}}\right) \sqrt{\tau(1+\tau)} G_{M_{p}}^{2} \tan ^{2} \frac{\theta_{e}}{2}
\end{gathered}
$$

where $I_{0} \propto G_{E_{p}}^{2}+\frac{\tau}{\varepsilon} G_{M_{p}}^{2}$. Measuring simultaneously these two components and taking their ratio gives the ratio of the form factors:

$$
\frac{G_{E p}}{G_{M p}}=-\frac{P_{t}}{P_{\ell}} \frac{\left(E_{e}+E_{e^{\prime}}\right)}{2 M_{p}} \tan \left(\frac{\theta_{e}}{2}\right)
$$

Neither the beam polarization nor the analyzing power of the polarimeter, used to measure $P_{t}$ and $P_{\ell}$, appear in Eqn. 4. 


\section{EXPERIMENTS}

In $1998 G_{E p} / G_{M p}$ was measured for $\mathrm{Q}^{2}$ from 0.5 to $3.5 \mathrm{GeV}^{2}$ [14]. Protons and electrons were detected in coincidence in the two high-resolution spectrometers (HRS) of Hall A. The polarization of the recoiling proton was measured in a graphite analyzer focal plane polarimeter (FPP). The data shown here differ from the previously published ones [14]; a reanalysis has been recently completed, taking into account the information from optical studies of both HRSs [? ], which eliminates the difficulty associated with the vanishing of the normal polarization component at the focal plane, when the precession angle in the dispersive plane is $180^{\circ}$ (or an integer multiple there of). Details of this reanalysis will appear in the archival paper in preparation [18]. In 2000 new measurements were made
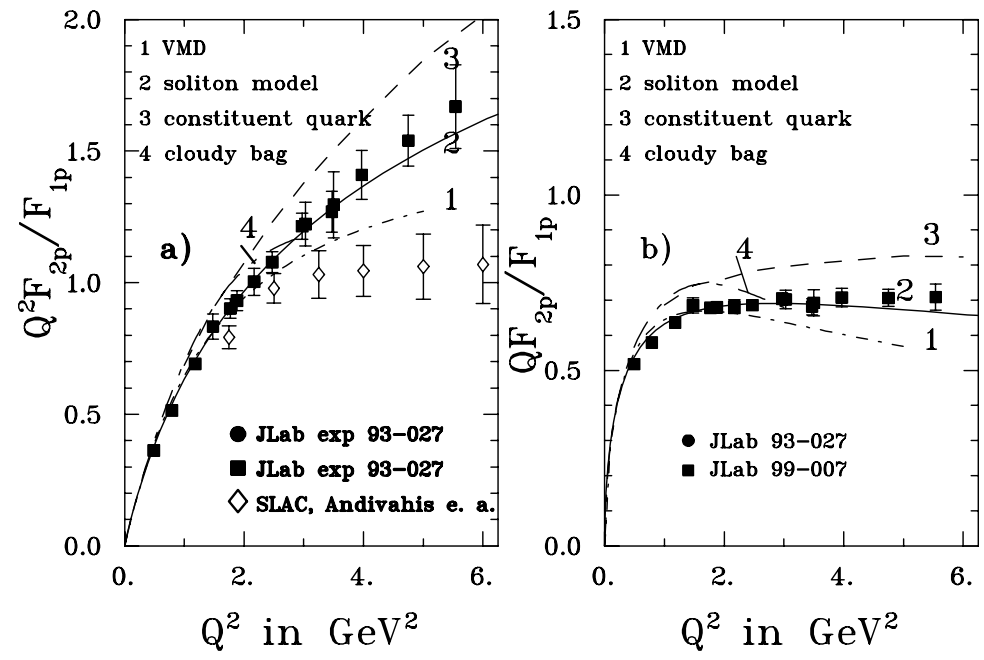

FIGURE 2. a) The ratio $Q^{2} F_{2 p} / F_{1 p}$ from the JLab experiments, compared with the data of ref. [12]. b) the ratio $Q F_{2 p} / F_{1 p}$ discussed in the text.

at $\mathrm{Q}^{2}=4.0,4.8$ and $5.6 \mathrm{GeV}^{2}$ with overlap points at $\mathrm{Q}^{2}=3.0$ and $3.5 \mathrm{GeV}^{2}$ [15]. To extend the measurement to these higher $\mathrm{Q}^{2}$, two changes were made. First, to increase the figure-of-merit of the FPP, a $\mathrm{CH}_{2}$ analyzer was used; the thickness was increased from $50 \mathrm{~cm}$ of graphite to $100 \mathrm{~cm}$ of $\mathrm{CH}_{2}\left(60 \mathrm{~cm}\right.$ for $\left.\mathrm{Q}^{2}=3.5 \mathrm{GeV}^{2}\right)$. Second, the electrons were detected in a lead-glass calorimeter with 9 columns and 17 rows of $15 \times 15 \times 35 \mathrm{~cm}^{3}$ blocks placed so as to achieve complete solid angle matching with the HRS detecting the proton. At the largest $\mathrm{Q}^{2}$ the solid angle of the calorimeter was 6 times that of the HRS. These data were analyzed with the new method mentioned above.

The combined results from both experiments are plotted in Fig. 1b) as the ratio $\mu_{p} G_{E p} / G_{M p}$. If the $\mu_{p} G_{E p} / G_{M p}$-ratio continues its linear decrease with the same slope, it will cross zero at $\mathrm{Q}^{2} \approx 7.5 \mathrm{GeV}^{2}$. In Fig. 1b), calculations based on VMD [4], a relativistic constituent quark (CQ) [2], and a soliton model[19] are shown. Also shown are results with another relativistic CQ model (rCQM) [3], with and without CQ form factor. Lomon [5] has reworked the Gary-Krumpelman VMD model [20] and obtains good agreement with the data for reasonable parameters for the vector-meson masses and coupling constants. 

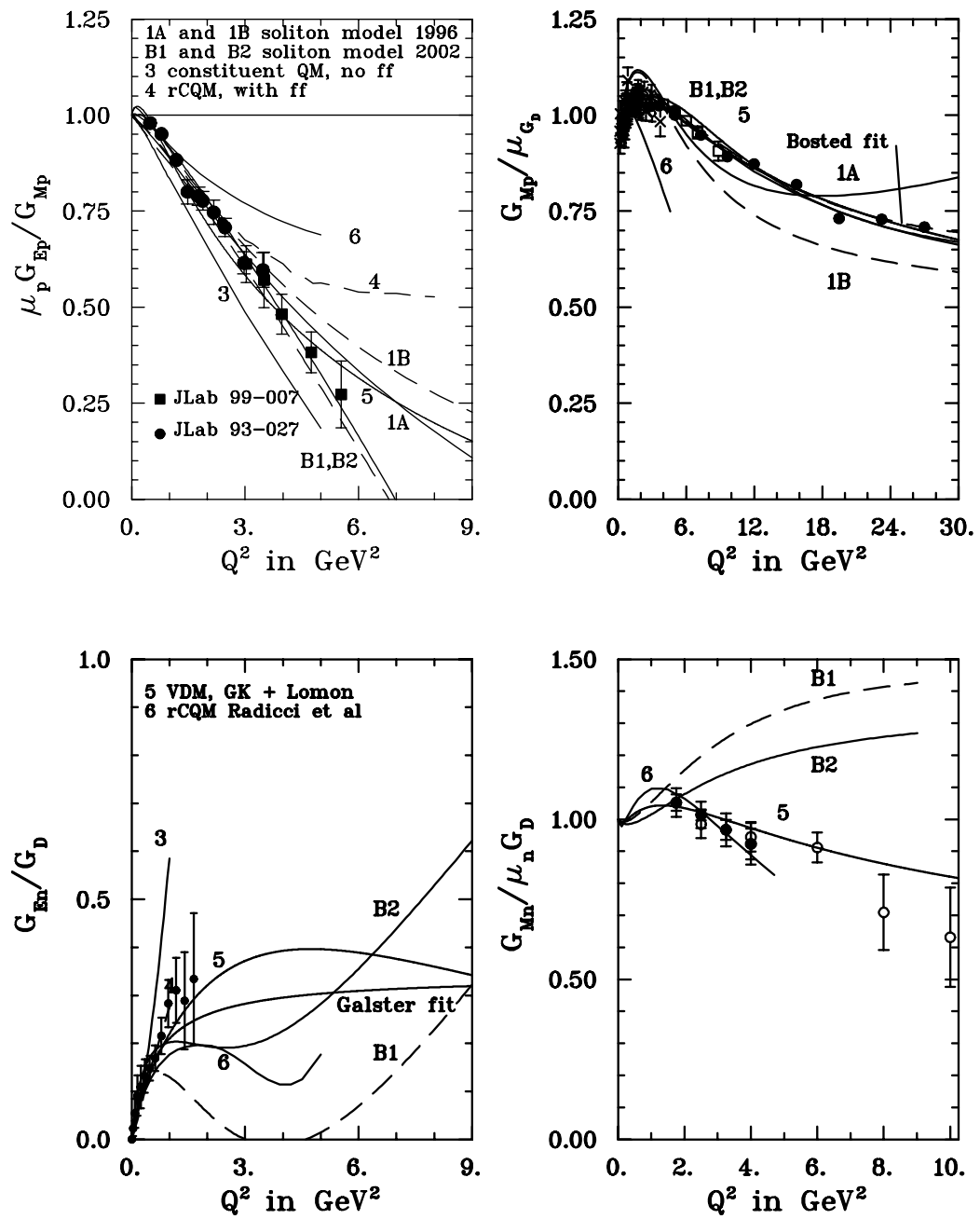

FIGURE 3. Theoretical predictions for $\mathrm{G}_{E}$ and $\mathrm{G}_{M}$ of the proton and neutron, along with selected data. For $\mathrm{G}_{E n}$ only the results of a recent analysis of elastic ed data from ref. [23] are shown; for $\mathrm{G}_{M n}$ only the larger $Q^{2}$ data of refs. [24] and [25] are shown. Curves labeled "Bosted fit" and "Galster fit" are from refs. [26] and [27], respectively.

\section{RESULTS AND DISCUSSION}

In Fig. 2a) the JLab data are shown as $\mathrm{Q}^{2}$ times $F_{2} / F_{1}$; pQCD predicts quenching of the spin flip form factor $F_{2}$, or equivalently helicity conservation; higher order contributions should make $Q^{2} F_{2} / F_{1}$ asymptotically constant. The data clearly contradict this prediction.

Shown in Fig. 2b) is $\mathrm{Q}$ times $F_{2} / F_{1}$, which reaches a constant value at $\mathrm{Q}^{2} \sim 2 \mathrm{GeV}^{2}$. Ralston et al. [21] have proposed that this scaling is due to the non-zero orbital angular momentum part of the proton quark wave function. Miller and Frank [22] have shown that imposing Poincare invariance leads to violation of the helicity conservation rule, and reproduces the $\mathrm{Q} F_{2} / F_{1}$ behavior. More demanding for models are predictions for all four form factors of the nucleon. The VMD fits are done in terms of the isoscalar 


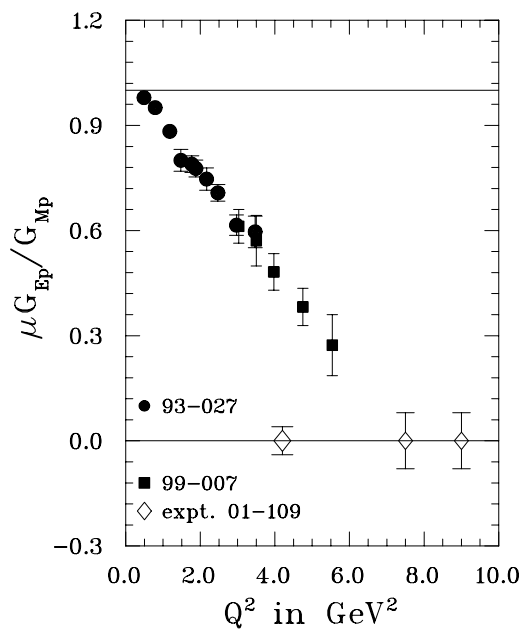

FIGURE 4. The expected statistical error bars for the two new data points at $\mathrm{Q}^{2}=7.5$ and $9 \mathrm{GeV}^{2}$, as well as a control point at $4.3 \mathrm{GeV}^{2}$; the systematcis uncertainties should be of the same size as shown in Fig. 1b).

FPP for 01-109 in Hall C

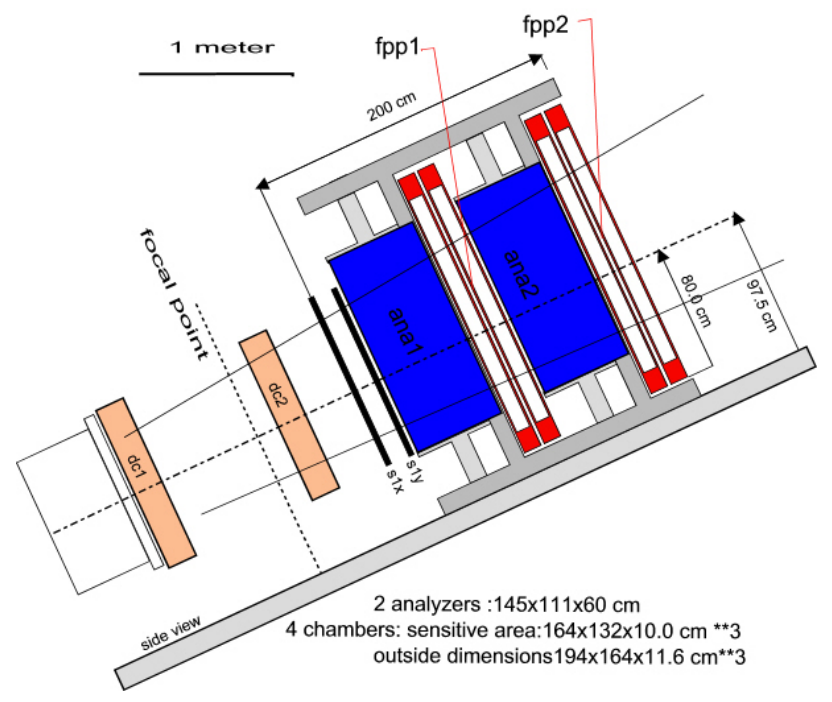

FIGURE 5. The new double focal plane polarimeter for the HMS in Hall C

and isovector form factors and thus naturally include all four form factors. In Fig. 3 predictions from the rCQM with SU(6) symmetry breaking [3], the soliton model [19], the point form model [29], and the VMD model of Ref. [5] are shown. The soliton model does well only for the proton. The recent VMD analysis [5] reproduces $G_{E p}, G_{M p}$ and $G_{M n}$ well, and predicts larger values for $G_{E n}$ than the fit of Ref. [27], in agreement with the preliminary data of Ref. [28]. 


\section{CONTINUATION TO $Q^{2}=9$ GEV $^{2}$}

The regular, quasi linear decrease of the $\mu_{p} G_{E p} / G_{M p}$ ratio with increasing $Q^{2}$ seen in Fig. 1b) suggests that the ratio may become zero. then negative at $\mathrm{Q}^{2}<10 \mathrm{GeV}^{2}$. Several theoretical prediction support this expectation. Accordingly, a proposal to continue these measurements to $\mathrm{Q}^{2}=9 \mathrm{GeV}^{2}$ was submitted to the JLab PAC in 2001. Following approval of this proposal, work has started on the construction of a new calorimeter as well as a new focal plane polarimeter to be installed in the Hall $\mathrm{C}$ high momentum spectrometer (HMS). The projected statistical errors for two news points as well as a control point at $4.3 \mathrm{GeV}^{2}$, are shown in Fig. 4. The electrons will be detected in a Cerenkov calorimeter with 1744 lead-glass bars of 45 (40) cm length, which is currently being built. The new polarimeter will consists of two polarimeters in series, each with a $\mathrm{CH}_{2}$ analyzer of 50-60 cm thickness to maximize the efficiency, as illustrated in Fig. 5. The analyzing power for $\mathrm{CH}_{2}$ has been recently measured in a calibration experiment at the JINR in Dubna, Russia. This calibration showed that the analyzing power for 5.3 $\mathrm{GeV} / \mathrm{c}$ protons was essentially constant up to a thickness of $80 \mathrm{~cm}$ of $\mathrm{CH}_{2}$ [30]; the proton momentum for $9 \mathrm{GeV}^{2}$ is $5.66 \mathrm{GeV} / \mathrm{c}$. However the efficiency stops increasing significantly with a $\mathrm{CH}_{2}$ thickness of about $60 \mathrm{~cm}$. The new experiment will be ready by the end of 2004.

\section{CONCLUSION}

The precise new JLab data on $\mu_{p} G_{E p} / G_{M p}$ show that this ratio continues to drop off linearly with increasing $\mathrm{Q}^{2}$ up to $5.6 \mathrm{GeV}^{2}$. The ratio $F_{2} / F_{1}$ does not follow the $1 / Q^{2}$ behavior predicted by $\mathrm{pQCD}$, and is a distinct signature of the non-perturbative regime dominating the $\mathrm{Q}^{2}$ range of the two JLab experiments described here. This behavior must be compared with the scaling of $Q^{4} G_{M} p$ seen in Ref. [6], which has been interpreted as indicative of $\mathrm{pQCD}$ for the magnetic form factor of the proton. Comparison of model calculations to the JLab data provides a stringent test of models of the nucleon.

\section{ACKNOWLEDGMENTS}

We thank our colleagues M. Jones, E. Brash, L. Pentchev and O. Gayou for their essential roles in the completion of these experiments. The Southeastern Universities Research Association manages the Thomas Jefferson National Accelerator Facility under DOE contract DE-AC05-84ER40150. U.S. National Science Foundation grant PHY 99 01182 supports my research.

\section{REFERENCES}

1. S.J. Brodsky and G.P. Lepage, Phys. Rev. D 22, (1981) 2157.

2. M.R. Frank, B.K. Jennings and G.A. Miller, Phys. Rev. C 54, 920 (1996). 
3. E. Pace, G. Salme, F. Cardarelli and S. Simula, Nucl. Phys. A 666\&667, 33c (2000). F. Cardarelli and S. Simula, Phys. Rev. C 62, 65201 (2000).

4. P. Mergell, U.G. Meissner, D. Drechsler Nucl. Phys. B A596, 367 (1996) ; and A.W. Hammer, U.G. Meissner and D. Drechsel, Phys. Lett. B 385, 343 (1996).

5. E. Lomon, Phys. Rev. C 64035204 (2001) and nucl-th/0203081.

6. A.F. Sill et al., Phys. Rev. D 48, 29 (1993).

7. J. Litt et al., Phys. Lett. B 31, 40 (1970).

8. Ch. Berger et al., Phys. Lett. B 35, 87 (1971).

9. L.E. Price et al., Phys. Rev. D 4, 45 (1971).

10. W. Bartel et al., Nucl. Phys. B 58, 429 (1973).

11. R.C. Walker et al, Phys. Rev. 49,5671 (1994).

12. L. Andivahis et al., Phys. Rev. D 50, 5491 (1994).

13. B. Milbrath et al, Phys. Rev. Lett. 80, 452 (1998); erratum Phys. Rev. Lett. 82, 221 (1999).

14. M. K. Jones et al., Phys. Rev. Lett. 84, 1398 (2000).

15. O. Gayou et al., Phys. Rev. Lett. 88092301 (2002)

16. A.I. Akhiezer and M.P. Rekalo, Sov. J. Part. Nucl. 3, 277 (1974); R. Arnold, C. Carlson and F. Gross, Phys. Rev. C 23, 363 (1981).

17. L. Pentchev, JLab Technical Note No/ TN-01-052 (200)

18. V. Punjabi et al., to be submitted to Physical Review C (2002)

19. G. Holzwarth, Z. Phys. A 356, 339 (1996) and private communication (2002).

20. M.F. Gari and W. Krumpelmann, Phys. Lett. B 274, 159 (1992).

21. J. Ralston et al., in Proc. of 7th International Conference on Intersection of Particle and Nuclear Physics, Quebec City (2000), p. 302, and private communication (2001).

22. G.A. Miller and M.R. Frank, nucl-th 0201021 (2002).

23. R. Schiavilla and I. Sick, nucl-ex/0107004 (2001).

24. A. Lung et al., Phys. Rev.Lett. 70, 718 (1993).

25. S. Rock et al. Phys. Rev. Lett. 49, 1139 (1982).

26. P. E. Bosted, Phys. Rev. 51, 409 (1995).

27. S. Galster et al. Nucl. Phys. B32, 221 (1971).

28. see R. Madey et al. to be published in the proceedings of the Elba Workshop "Electron-nucleus Scattering VII", and contribution to this conference (B. Plaster et al.)

29. R.F. Wagenbrunn, S. Boffi, W.H. Klink, W. Plessas and M. Radici, Phys. Lett. B, 511:33 (2001).

30. L.S. Azghirey et al, report to this conference and to be published (2002) 